\title{
Enhanced Fingernail Recognition based on GLCM, SIFT and NN
}

\author{
Silky \\ M.Tech Scholar \\ Department of Computer Science, \\ Shaheed Bhagat Singh State Technical Campus, \\ Ferozepur
}

\author{
Sonika Jindal \\ Assistant Professor \\ Department of Computer Science, \\ Shaheed Bhagat Singh State Technical Campus, \\ Ferozepur
}

\begin{abstract}
Biometric validation innovations, for example, unique mark, face, iris, and vein, have been across the board in numerous applications from singular convenient gadgets to national ID administration frameworks. Besides, moderately more up to date biometric validation modalities including eye development, lip-movement, and so on, have been additionally looked into and created keeping in mind the end goal to enhance confirmation exactness and an ease of use of a biometric verification framework. What's more, some these sorts of modalities are powerful as a liveness identification strategy, which cannot just enhance verification exactness in customary biometric confirmation frameworks, yet additionally diminish dangers with respect to hostile to mocking assaults. This paper propose a GSN (GLCM, SIFT, $\mathrm{NN}$ ) based finger nail recognition technique. GLCM is used for feature extraction; SIFT for Key-point extraction and NN for recognition.
\end{abstract}

\section{Keywords}

Biometric Authentication, Finger Nail Recognition, GLCM, SIFT, NN, Feature Extraction.

\section{INTRODUCTION}

Image processing assumes an imperative part in highlight extraction that change over a picture into advanced shape by playing out a few activities on it for separating learned data from it. The organic significance of the biometric word is life - estimations. In this physical normal for a man that can be kept an eye on a robotized premise which is utilized for security purposes and access control applications. Transient biometric can be utilized as a part of genuine application for distinguishing proof and security thought processes. For this surface highlights separated from the finger nail plate. Transient biometric is another idea and it's for brief time and furthermore database will change inside the a few months ${ }^{\text {ee }}$ interim. Biometrics is the computerized distinguishing proof of people in view of their physical attributes. There is no compelling reason to recollect any passwords or cards utilizing the transient biometric framework and there is less danger of abuse or overlooked the information. Biometrics frameworks work by recording and looking at biometric attributes. At the point when an individual first uses a biometric framework, their distinguishing highlights are enlisted as a source of perspective for future examination.
The nail is a skin limb arranged at the dorsal surface of the finger. It fundamentally comprises of the nail plate and firmly weaved keratinized layers that is nail quaint little inn framework. The nail bed is the skin underneath the nail surface. It is made of two kinds of tissues: the more profound dermis, which is the living layer settled deep down, and the shallow epidermis, layer just underneath the nail plate which pushes ahead with the nail plate. These epidermis edges lie between parallel dermis papillae in a tongue-in-groove mold and structures a remarkable parallel structure that is copied on the external surface of the nail as longitudinal striations. Accordingly the independence in the uniqueness of nail construct biometrics is very needy in light of the inherent anatomic qualities of the nail [2].

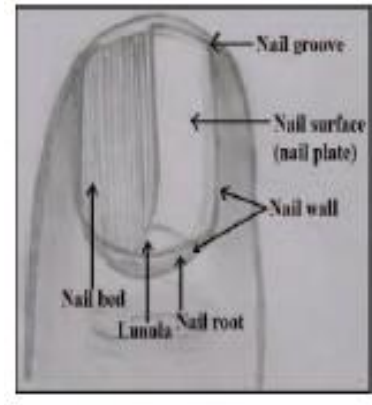

(a)

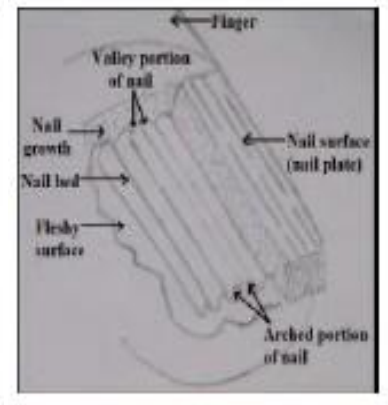

(b)
Fig 1: (a) Human finger nail (b) Magnification of the structure beneath the nail surface [2]

\section{BACKGROUND}

This area speaks to the writing study done on the different surfaces include extraction, coordinating, characterization systems. The writing survey speaks to the work done by the different specialists and researchers toward this path which fills in as a prologue to explore assist on different strides of enhancing thruways security. Barbosa et al. presented another idea for biometric acknowledgment called Transient biometrics. conventional point of view of biometric acknowledgment frameworks focuses on biometric attributes that are as steady as could be expected under the circumstances, (for example, the eye retina), giving precision after some time however in the meantime bringing about protection from their utilization for non-basic applications because of the likelihood of abuse. Interestingly, transient biometrics depends on biometric attributes that do change after some time going for expanded acknowledgment in non- 
basic applications [1]. Garg et al. displayed another biometric framework in view of the external surface of the finger nail. There has not been any endeavor fit as a fiddle and surface for human confirmation. The nail bed data, imitated on the mail surface ends up being an extremely special and stable biometric identifier for individual confirmation. Be that as it may, examine writing presents some unpredictable set-up and the utilization of interferometer strategy for extraction of nail bed subtle elements. This work proposed an extremely basic and helpful strategy to portion nail surface from the client hand and utilize it as a biometric identifier [2].Easwaramoorthy et al. exhibited a novel biometric identifier utilizing finger nail surfaces. The structure of nail surface is thought to be very exceptional, even if there should be an occurrence of two indistinguishable twins and between various finger nails of a person. In this work exertion are made to explore the genuine capacities and execution that can be accomplished through single finger nail surface for confirmation. Scale Invariant Feature Transform with Support Vector Machine as a classifier is given high exactness for finger nail verification [3].

Devi et al. investigated the current research works identified with nail plate and nail framework as device for bio-metric framework, nail crease vessels to distinguish the malady seriousness levels and influenced organs, nail surface as confirmation in scientific science to recognize the synthetic impacts, nail tests to distinguish tranquilize admission and manhandle and so forth. So that, Nail is dissected by different imaging writes and preparing calculations to perceive the individual's uniqueness, wellbeing condition and its history. This paper likewise distinguished the exploration difficulties and issues [4]. Jain et al. exhibited a prologue to biometric acknowledgment. A wide assortment of frameworks requires solid individual acknowledgment plans to either affirm or decide the personality of an individual asking for their administrations. The motivation behind such plans is to guarantee that the rendered administrations are gotten to just by a real client and nobody else. Cases of such applications incorporate secure access to structures, PC frameworks, PCs, mobile phones, and ATMs [5]. Phillips et al. considered two promising picture based biometrics, faces and fingerprints. This paper exhibited a basic evaluation of the best in class, recommend future research bearings, and recognize innovative difficulties [6].

Abe et al. presented moderately fresher imminent biometric confirmation modalities with a specific end goal to use as a supplemental methodology or to understand a ceaseless verification framework. Furthermore, a few papers say that a few modalities, for example, eye development, lip-movement, and cardiovascular data, are viable as a liveness location strategy, which cannot just enhance validation precision in customary biometric verification frameworks, yet in addition diminish dangers with respect to against satirizing assaults [7]. Kureel et al. proposed a novel plan for palmprint acknowledgment utilizing a shape and Texture based component investigation acquired from Statistical Image Features. The palmprint picture is portrayed by a rich arrangement of highlights including central lines, edges, and wrinkles. Hence, the utilization of a suitable surface descriptor conspire is required to catch this data exact [8]. Barbosa et al. talked about 'transient biometrics,' i.e. acknowledgment through biometric attributes that will change temporarily and demonstrate that pictures of the fingernail plate can be utilized as a transient biometric with a valuable life expectancy of under a half year. An immediate approach is recommended that requires no preparation and a significant assessment dataset is made freely accessible [9].

\section{PROPOSED TECHNIQUE}

The structure of the nail surface is thought to be very one of a kind, even on account of two indistinguishable twins or even between various finger nails of a person. The nearness of various keratin writes in various areas of the nail network and nail bed might be in charge of the variable physical qualities of the people nail surface. One of the key factors that is refered to for the inclination of nail surface based biometric is that the nail bed is secured and covered up by the nail surface altogether diminishing the catch and use of this biometric in ridiculing instrument. Additionally, the hand database is effectively collectable and the little size of the nail locale extricated from the hand tests, takes less calculation time, expanding its reasonableness for continuous applications. The objectives of this research work is as follows:

- Study and Analyse the different biometric techniques used for fingernail Image Recognition.

- Apply GSN (GLCM, SIFT and NN) to Evaluate features, Key-points and Perform Recognition.

- $\quad$ Find the Final Matched Image from Database.

The methodology of the proposed technique is as follows:

- Input Image from finger nail Database.

- Convert Image into BW.

- Apply GLCM for feature selection.

- Create Histogram of features.

- Apply SIFT on Image by extraction key points and perform matching to find similar finger nail picture.

- Perform NN Training.

- Perform NN Testing.

- Find Matched Image.

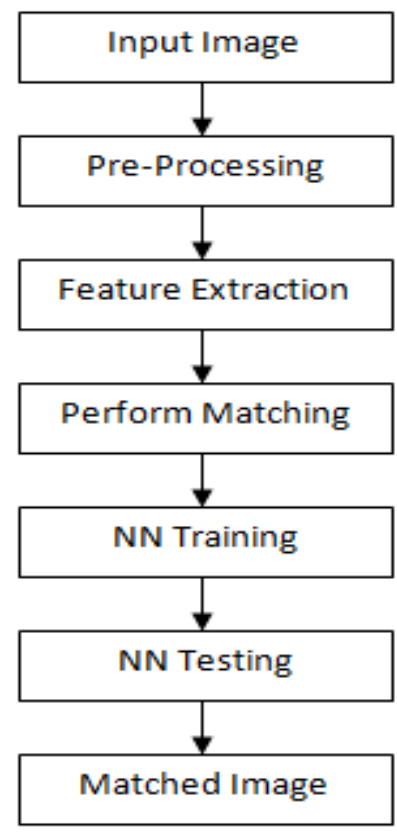

Fig 2: Proposed Technique

Gray-Level Co-occurrence Matrix: A factual strategy for looking at surface that considers the spatial relationship of pixels is the gray-level co-occurrence network (GLCM), otherwise called the dim level spatial reliance grid. The 
GLCM capacities portray the surface of a picture by ascertaining how frequently combines of pixel with particular esteems and in a predetermined spatial relationship happen in a picture, making a GLCM, and after that separating factual measures from this lattice.

Scale Invarient Feature Transform: It is a system to recognize notable, stable feature points in a picture. For each such point, it likewise gives an arrangement of "highlights" that "portray/depict" a little picture district around the point. These highlights are invariant to pivot and scale. It consists of various steps:

- Scale - Space Extrema Detection

- Keypoint Localization

- Orientation Assignment

- Keypoint Descriptor

- Keypoint Matching

Neural Network: In Neural Network we first perform the training of all features, and then we do matching the results as per testing image. When we are done with Training, it will give us Net file of neural network to perform testing.

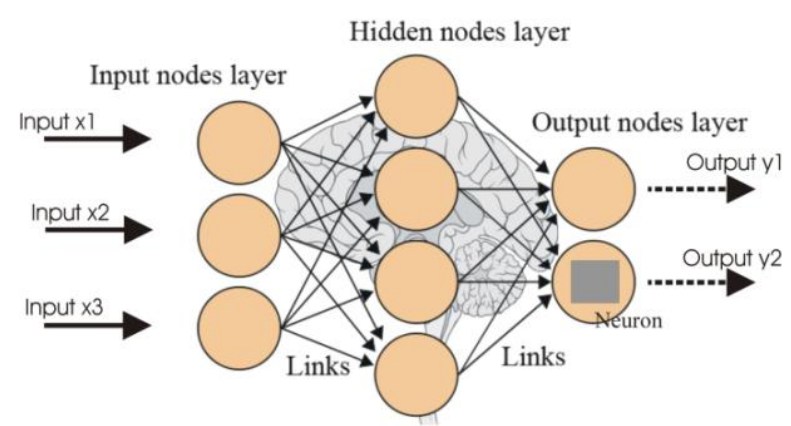

Fig 3: Neural Network

\section{PROPOSED TECHNIQUE}

The proposed technique is implemented using MATLAB tool. This section presents the results of the proposed technique. Proposed method is tested on various types of finger nail plate images with different orientations and magnitudes of the texture. The figure below shows the finger nail image database.

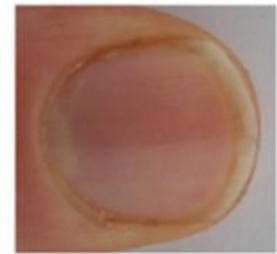

(a)

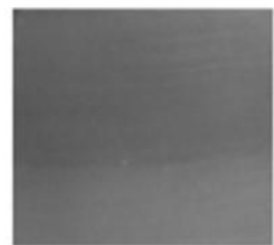

(d)

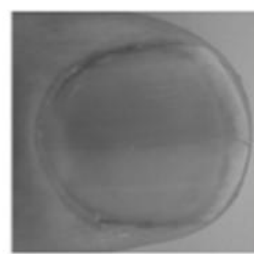

(b)

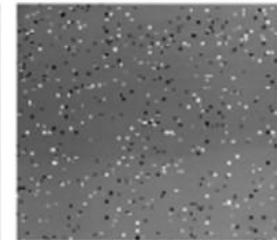

(e)

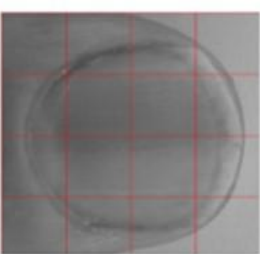

(c)

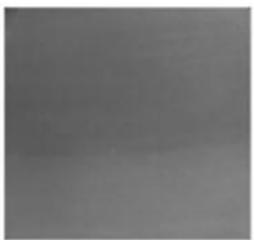

(f)
Fig 4: Pre-processing Results
Selected image

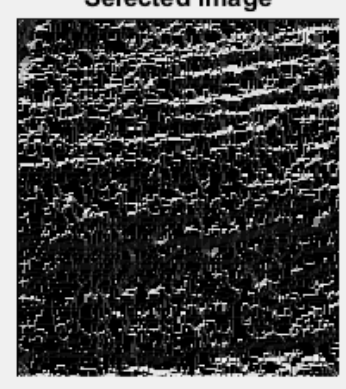

Image with key points mapped onto it

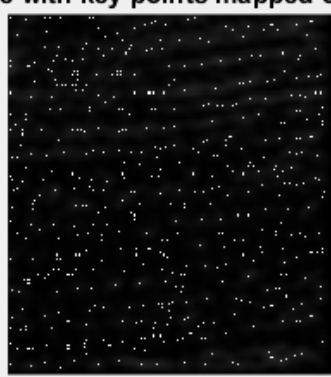

Fig 5: Selected Image and Image with Key Points
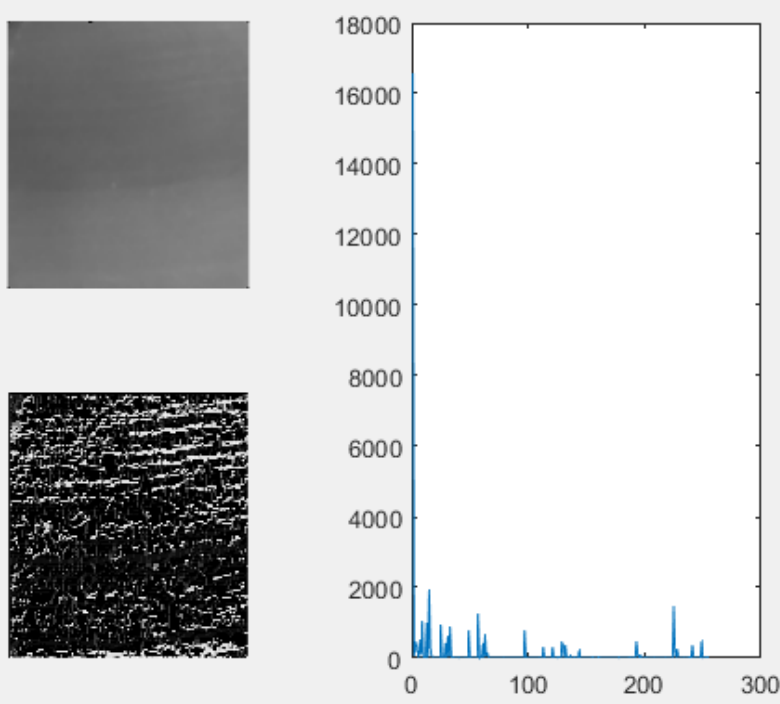

Fig 6: Histogram of Selected Image 


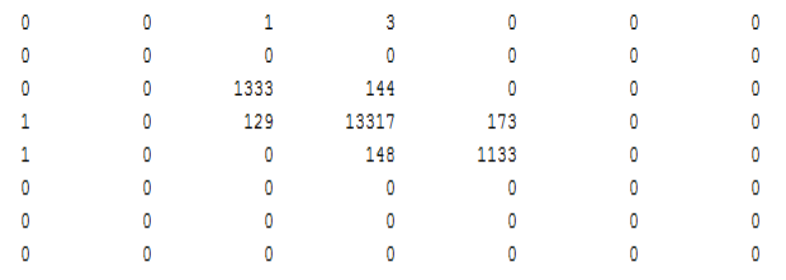

Fig 7: Feature Extraction with GLCM

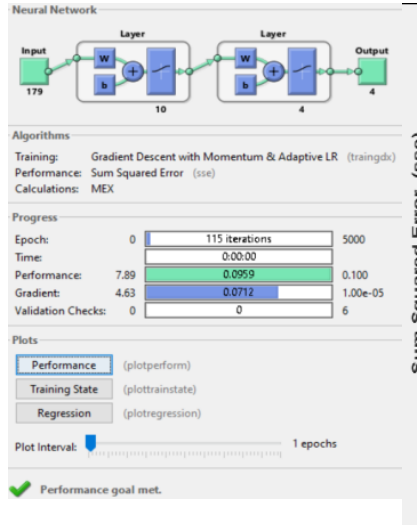

(a)

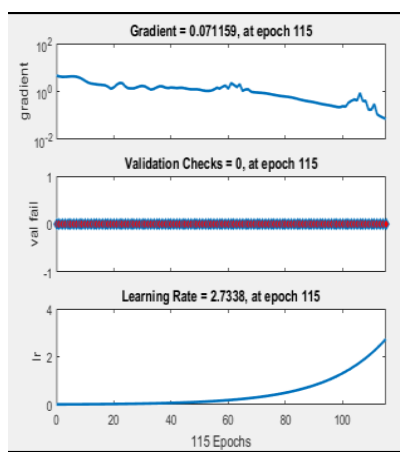

(c)

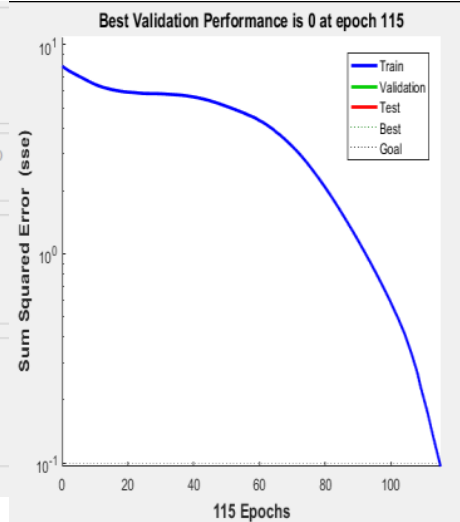

(b)

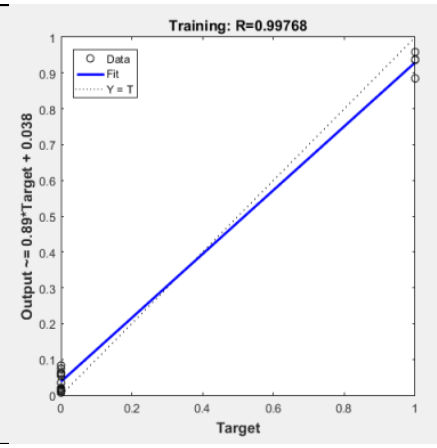

(d)
Fig 8: (a) Neural Network (b) Best Validation

Performance of Neural Network at 115 Epochs (c)

Gradient, Validation Checks and Learning Rate of Neural Network at 115 Epochs (d) Training Rate

Table 1: Showing Matching Percentage and Testing Time of Various Nail Images

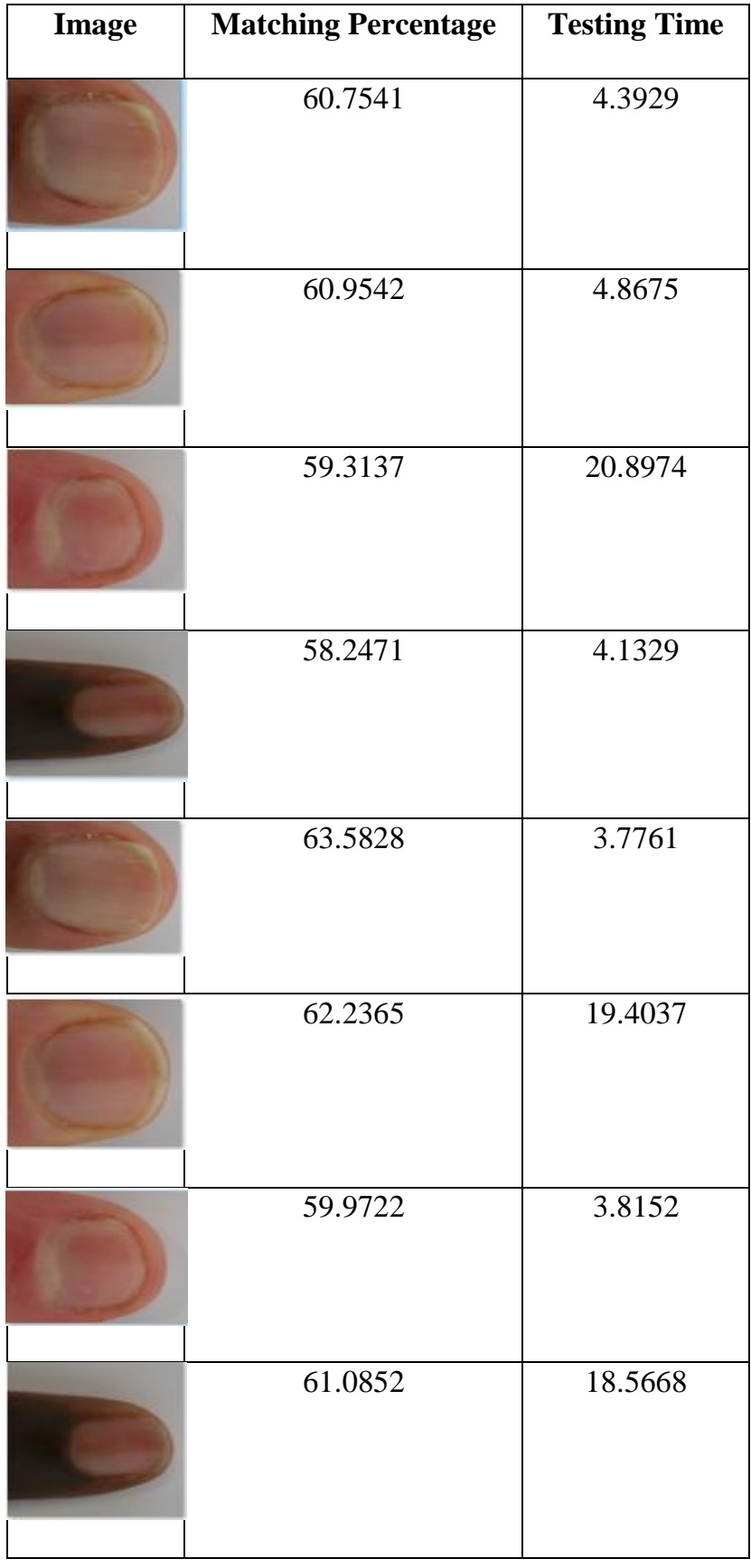

\section{CONCLUSION}

This paper propose a GSN (GLCM, SIFT, NN) based finger nail recognition technique. Various biometric techniques used for fingernail recognition are analyzed. GLCM is used to extract features, SIFT technique is applied then to extract keypoint and finally recognition is performed by using NN training and testing. The technique is implemented in MATLAB. Experimental results demonstrate that the proposed technique performs recognition in an efficient manner. 


\section{REFERENCES}

[1] Igor Barros Barbosa, Theoharis Theoharis, Christian Schellewald, Cham Athwal, "Transient Biometrics using Finger Nails".

[2] Shruti Garg, Amioy Kumar, M. Hanmandlu, "Biometric Authentication Using Finger Nail Surface", IEEE 12th International Conference on Intelligent Systems Design and Applications (ISDA), 2012, pp. 497-502.

[3] Sathishkumar Easwaramoorthy, Sophia F, Prathik A, "Biometric Authentication using finger nails", IEEE, 2016.

[4] Dr. M. Renuka Devi, Thahira Banu.V, "Study of Nail Unit using Image Processing Methods", IEEE International Conference on Computer Communication and Informatics, 2015.

[5] Anil K. Jain, Arun Ross, Salil Prabhakar, "An Introduction to Biometric Recognition", IEEE Transactions on Circuits And Systems for Video Technology, Vol. 14, NO. 1, 2004, pp. 4-20.

[6] P. Jonathon Phillips, RMichael McCabe, Rama Chellappa, "Biometric Image Processing and Recognition".

[7] Narishige Abe, Takashi Shinzaki, "A Survey on Newer Prospective Biometric Authentication
Modalities”, Josai Mathematical Monographs vol. 7 (2014), pp. 25-40.

[8] Srushti Kureel, Praveen Kumar, "Shape and Texture based Palm Print Recognition System for Biometric identification “,International Journal of Engineering Trends and Technology (IJETT) - Volume 50 Number 1 August 2017 , pp. 39-44.

[9] Igor Barros Barbosa, Theoharis Theoharis, Ali E. Abdallah, "On the use of fingernail images as transient biometric identifiers", 2016.

[10] R. V. Hogg, A. T. C, "Introduction to Mathematical Statistics", New York: Macmillan, IEEE, 1965.

[11] D. N. Graham, "Image transmission by twodimensional contour coding", vol. 55, IEEE, 1967, pp. 336-346.

[12] Robert M. Harlick, K. S, "Textural Feature for Image Classification”, IEEE, 1973.

[13] A.H. Mir, "Texture analysis of CT images, Engineering in Medicine and Biology NovemberDecember”, IEEE, 1995.

[14] T. Ojala, M. P., "Gray Scale and Rotation Invariant Classification with Local Binary Pattern”, 1997. 